Pacific Journal of Mathematic 


\title{
TANGENT WINDING NUMBERS AND BRANCHED MAPPINGS
}

\author{
J. R. QUINE
}

The notion of tangent winding number of a regular closed curve on a compact 2-manifold $M$ is investigated, and related to the notion of obstruction to regular homotopy. The approach is via oriented intersection theory. For $N$, a 2-manifold with boundary and $F: N \rightarrow M$ a smooth branched mapping, a theorem is proved relating the total branch point multiplicity of $F$ and the tangent winding number of $\left.F\right|_{\partial_{N}}$. The theorem is a generalization of the classical RiemannHurwitz theorem.

1. Introduction. Let $M$ be a smooth, connected, oriented 2manifold and let $f$ and $g$ be regular closed curves on $M$ with the same initial point and tangent direction. An integer obstruction to regular homotopy $\gamma(f, g)$ is derived which is uniquely defined if $M \neq S^{2}$ and defined $\bmod 2$ if $M=S^{2}$. Let $F(t, \theta)$ be any homotopy such that $F(0, \theta)=f(\theta)$ and $F(1, \theta)=g(\theta)$ and $F$ is smooth on the interior of the unit square. It is shown that $\gamma(f, g)=I\left(\partial F / \partial \theta, M_{0}\right)$, where $M_{0}$ is the zero section as a sub-manifold of $T M$, and $I$ denotes the total number of oriented intersections. This is given interpretation as the number of loops acquired by curves $F(t)=,f_{t}$ in homotopy.

If $M$ is compact and $y$ is not on the image of $f$, then we define twn $(f ; y)$, a generalization of the tangent winding number. We show that $\gamma(f, g)=\operatorname{twn}(g ; y)-\operatorname{twn}(f ; y)+I(F, y) \chi(M)$, where is the Euler characteristic. If $N$ is a 2-manifold with boundary and $F: N \rightarrow M$ is a smooth branched mapping and $\partial F=\left.F\right|_{\partial N}$, we show that $\operatorname{twn}(\partial F ; y)+I(F, y) \chi(M)=\chi(N)+r$, where $r$ is the total branchpoint multiplicity and $y$ is not in $F(\partial N)$. We show that the Riemann-Hurwitz theorem follows as a corrollary.

2. The obstruction to regular homotopy. Let $M$ be a smooth, connected 2-manifold with Riemannian metric. Let $T M$ be the tangent bundle and $\widetilde{T} M$ the unit tangent or sphere bundle. Let $f: R \rightarrow M$ with $f(\theta)=f(\theta+1)$ for all $\theta \in R$ be a regular closed curve on $M$, that is, $f$ has continuously turning, nonzero tangent vector at each point. Given $F:[0,1] \times R \rightarrow M$ continuous with $F(t, \theta)=F(t, \theta+1)$ for all $\theta \in R$, then $F$ is said to be a regular homotopy if each closed curve $F(t$,$) is regular for 0 \leqq t \leqq 1$. We say the curves $f(\theta)=F(0, \theta)$ and $g(\theta)=F(1, \theta)$ are regularly homo- 
topic.

Suppose now that $f$ and $g$ are regular closed curves with $f(0)=g(0)=y_{0}$. Let $\widetilde{f}$ and $\widetilde{g}$ be the closed curves on $\widetilde{T} M$ obtained by taking the unit tangent vector at each point of $f$ and $g$ respectively. Suppose that $\widetilde{f}(0)=\widetilde{g}(0)=\widetilde{y}_{0}$. Smale [9] has shown that $f$ and $g$ are regularly homotopic iff $\widetilde{f}$ and $\widetilde{g}$ are homotopic. Using this result we define the obstruction to regular homotopy, $\gamma(f, g)$, as follows.

Let $S^{1}$ be the fiber of $\widetilde{T} M$ over $y_{0}$. Since $\Pi_{2}(\widetilde{T} M)=0$ for any 2-manifold $M$ (this is clear if $\Pi_{2}(M)=0$ and can be verified directly if $M$ is $S^{2}$ or the projective plane), we have the following portion of the exact homotopy sequence of the bundle $\widetilde{T} M$ over $M$

$$
0 \longrightarrow \Pi_{2}(M) \stackrel{\phi}{\longrightarrow} \Pi_{1}\left(S^{1}\right) \stackrel{\mu}{\longrightarrow} \Pi_{1}(\widetilde{T} M) \stackrel{\psi}{\longrightarrow} \Pi_{1}(M) \text {. }
$$

The sequence (1) induces an isomorphism

$$
j: \operatorname{ker} \psi \longrightarrow \Pi_{1}\left(S^{1}\right) / \operatorname{im} \phi .
$$

If $f$ and $g$ are homotopic, then the product $[\widetilde{g}][\widetilde{f}]^{-1}$ is in ker $\psi$. Writing $\alpha=j\left([\widetilde{g}][\tilde{f}]^{-1}\right)$, Smale's theorem says that $f$ and $g$ are regularly homotopic iff $\alpha=0$.

Now in what follows suppose $M$ is oriented. This gives us a natural choice of orientation on $S^{1}$ as the fiber of $\widetilde{T} M$ at $y_{0}$, which in turn determines a "positively oriented" generator of $\Pi_{1}\left(S^{1}\right)$. This generator determines an isomorphism of $\Pi_{1}\left(S^{1}\right)$ with the integers $Z$. Now $\Pi_{2}(M)=0$ unless $M=S^{2}$. Identifying $\Pi_{1}\left(S^{1}\right)$ with $Z$, we see that $\operatorname{im} \phi=2 Z$ in case $M=S^{2}$. (Since the Euler characteristic of $S^{2}$ is 2 , the fundamental 2-cycle is mapped into 2 by $\varnothing$.) Thus for $M \neq S^{\prime}, \alpha$ is an integer which we denote $\gamma(f, g)$. If $M=S^{2}, \alpha$ is an element of $Z_{2}$. In this case we write $n=\gamma(f, g)$ if the integer $n$ determines the class $\alpha$ in $Z_{2}$. We will refer to $\gamma(f, g)$ as the obstruction to regular homotopy. We remark that $\gamma(f, g)$ is only defined if $f$ and $g$ are homotopic. In the next section we will show how to characterize $\gamma(f, g)$ using intersection theory and in a later section we explain its relationship to tangent winding numbers on surfaces as in Reinhart [8] and Chillingworth [1].

3. A characterization of $\gamma(f, g)$. Let $f, g$ and $M$ be as in the previous section. We will continue to assume that $M$ is oriented. Suppose $F(\theta, t)$ is a homotopy, not necessarily regular, with $F(0, \theta)=$ $f(\theta)$ and $F(1, \theta)=g(\theta)$ for all $\theta$. Let $K$ be the square $[0,1] \times[0,1]$ and write $F: K \rightarrow M$. Now the pullback bundle $F^{*}(T M)$ is trivial over $K$, so we can find vector valued functions $v_{1}, v_{2}: K \rightarrow T M$ such that the ordered pair $\left(v_{1}(x), v_{2}(x)\right)$ is positively oriented in $T M_{F(x)}$ 
for all $x \in K$. Now consider $\partial / \partial \theta$ as a section of $T K$ and write $F_{*} \circ(\partial / \partial \theta)=(\partial F / \partial \theta): K \rightarrow T M$. Write $(\partial F / \partial \theta)(x)=p_{1}(x) v_{1}(x)+p_{2}(x) v_{2}(x)$ where $p=\left(p_{1}, p_{2}\right): K \rightarrow R^{2}$. By the definition of the map $\mu$ in the exact sequence (1), we see that the preimage of $[\widetilde{g}][\tilde{f}]^{-1}$ under is just $\left.\operatorname{deg}(p /|p|)\right|_{\partial K}$, where $\partial K$ is the positively oriented boundary of $K,||$ is the usual Euclidean norm in $R^{2}$, and deg is topological degree. Thus $\gamma(f, g)=\left.\operatorname{deg}(p /|p|)\right|_{\partial K}$. If $M=R^{2}$ and $v_{1}=(1,0)$, $v_{2}=(0,1)$ then $\gamma(f, g)=\operatorname{twn} g-\operatorname{twn} f$, where twn denotes tangent winding number.

Now suppose $x$ is an isolated zero of $\partial F / \partial \theta$ and $D$ is a closed coordinate disc containing $x$, but no other zeros of $\partial F / \partial \theta$. We define

$$
\operatorname{ind}_{x} \frac{\partial F}{\partial \theta}=\left.\operatorname{deg} \frac{p}{|p|}\right|_{\partial D} .
$$

This is easily verified to be independent of the choice of $v_{1}$ and $v_{2}$. Thus if all the zeros of $\partial F / \partial \theta$ are isolated, then

$$
\gamma(f, g)=\sum_{x \in S} \operatorname{ind}_{x} \frac{\partial F}{\partial \theta}
$$

where $S$ is the set of zeros of $\partial F / \partial \theta$.

Now suppose that $F$ is smooth on int $K$, and let $M_{0}$ be the zero section of $T M$ considered as a smooth, oriented 2-submanifold of $T M$. If $\partial F / \partial \theta$ intersects $M_{0}$ transversely at $x \in K$, then $\operatorname{ind}_{x} \partial F / \partial \theta$ is the same as the oriented intersection number of $\partial F / \partial \theta$ with $M_{0}$ at $x$. (For an explanation of intersection numbers see Guillemin and Pollack [3].) Thus $\gamma(f, g)=I\left(\partial F / \partial \theta, M_{0}\right)$, the total number of oriented intersections of $\partial F / \partial \theta$ with $M_{0}$. We remark that $I\left(\partial F / \partial \theta, M_{0}\right)$ is defined even if $\partial F / \partial \theta$ does not intersect $M_{0}$ transversely: we simply count the transverse intersections for a "nearby" map. Since $\partial F / \partial \theta(\partial K) \cap M_{0}=\varnothing$, the total number of intersections is the same for every "nearby" map. We summarize our results in

THEOREM 1. Let $f$ and $g$ be regular closed curves on $M$ with the same initial points and initial tangent directions. Suppose $f$ and $g$ are homotopic and $F: K \rightarrow M$ is a homotopy, smooth on int $K$, with $F(0, \theta)=f(\theta)$ and $F(1, \theta)=g(\theta)$, then the obstruction to regular homotopy $\gamma(f, g)$ is equal to $I\left(\partial F / \partial \theta, M_{0}\right)$, the total number of oriented intersections of $\partial F / \partial \theta$ with the zero section $M_{0}$.

We give the following interpretation of Theorem 1. Suppose $\partial F / \partial \theta(x)=0$ where $x=\left(t_{0}, \theta_{0}\right)$ and suppose $\partial F / \partial \theta$ intersects $M_{0}$ transversely at $x$. The curve $F\left(t_{0}, \theta\right)$ has a cusp at $\theta=\theta_{0}$. As $t$ increases, if this cusp represents the appearance of a positively oriented 
loop or the disappearance of a negatively oriented loop, then the intersection number at $x$ is 1 . If it represents the appearance of a negatively oriented loop or the disappearance of a positively oriented loop, then the intersection number is -1 . Thus $I\left(\partial F / \partial \theta, M_{0}\right)$ counts the null homotopic loops lost or gained in the homotopy.

4. Tangent winding numbers. We now wish to show the relationship between $\gamma(f, g)$ as defined in the previous section and the notion of tangent winding number of a regular curve with respect to a vector field $v$ on a compact 2-manifold $M$ as in Reinhart [8] and Chillingworth [1]. Suppose $f$ is a regular closed curve on $M$ and $v$ is a vector field on $M$ which vanishes at a single point $y$ not on the image of $f$. The order that $v$ vanishes at $y$ is clearly $\chi(M)$. We define $\operatorname{twn}_{v} f$ to be the number of times the tangent of $f$ rotates in relation to $v$. More specifically, suppose $v=v_{1}$ and choose vector field $v_{2}$ such that $\left(v_{1}, v_{2}\right)$ is a positively oriented basis except at $y$, where both vanish to the order $\chi(M)$. Write $d f / d \theta=$ $p_{1} v_{1}+p_{2} v_{2}$ where $p=\left(p_{1}, p_{2}\right): S^{1} \rightarrow R^{2}$. We then define $\operatorname{twn}_{v} f$ to be $\operatorname{deg} p /|p|$. It is straightforward to show that $\operatorname{twn}_{v} f$ depends only upon the choice of $y$, in fact, it depends only upon the component of $M-f(R)$ in which $y$ lies. Thus, we write $\operatorname{twn}(f ; y)$ in place of $\operatorname{twn}_{v} f$.

THEOREM 2. Suppose $M$ is compact and let $f, g$, and $F$ be as in Theorem 1. Let $y \in M-f(R) \cup g(R)$, then $\gamma(f, g)=I\left(\partial F / \partial \theta, M_{0}\right)=$ twn $(g ; y)-\operatorname{twn}(f ; y)+I(F, y) \chi(M)$.

Proof. Let $v_{1}$ and $v_{2}$ be as in the definition of twn $(f ; y)$. Without loss of generality, suppose $y$ is a regular value of $F$, $(\partial F / \partial \theta) \neq 0$ on $F^{-1}(y)$, and $\partial F / \partial \theta$ has only isolated zeros. Let $x_{1}, \cdots, x_{m}$ be the zeros of $\partial F / \partial \theta$ and $\left\{x_{m+1}, \cdots, x_{l}\right\}=F^{-1}(y)$. Write $(\partial F / \partial \theta)(x)=q_{1}(x) v_{1}(F(x))+q_{2}(x) v_{2}(F(x))$ for $x \notin F^{-1}(y)$. Let $T_{1}, \cdots, T_{l}$ be closed disjoint coordinate discs on $M$ such that $x_{k} \in T_{k}$ for $k=$ $1, \cdots, l$. Since $v_{1}$ and $v_{2}$ vanish of order $\chi(M)$ at $y$, we have

(a) For $k=m+1, \cdots,\left.\operatorname{deg}(p /|p|)\right|_{\partial T_{k}}= \pm \chi(M)$ where the sign is negative if $F$ preserves orientation at $x_{k}$, and positive if $F$ reverses orientation at $x_{k}$.

(b) For $k=1, \cdots, m,\left.\operatorname{deg}(p /|p|)\right|_{\partial T_{k}}=\operatorname{ind}_{x_{k}}(\partial F / \partial \theta)$.

Now since $p: K-\bigcup_{k=1}^{m} T_{k} \rightarrow R^{2}$, we have that

$$
\left.\operatorname{deg}(p /|p|)\right|_{\partial K}=\left.\sum_{k=1}^{m} \operatorname{deg}(p /|p|)\right|_{\partial T_{k}} .
$$

Since by definition $\left.\operatorname{deg}(p /|p|)\right|_{\partial K}=\operatorname{twn}(g ; y)-\operatorname{twn}(f ; y)$, the theorem follows from Remarks (a) and (b). 
Thus we see that $\operatorname{twn}(g ; y)-\operatorname{twn}(f ; y)$ determines $\bmod \chi(M)$ the obstruction to regular homotopy.

5. Branched mappings. Let $\tilde{N}$ be a compact oriented 2-manifold and let $D_{1}, \cdots, D_{n}$ be $n$ disjoint copies of the closed unit disc on $\tilde{N}$. Let

$$
N=\tilde{N}-\bigcup_{k=1}^{n} \operatorname{int} D_{k}
$$

Let $M$ be a compact oriented 2-manifold. Let $F: N \rightarrow M$ be smooth. Say $F$ is a branched mapping if $F$ is nonsingular and orientation preserving except at a finite number of points in int $N$ where $F$ behaves locally like the complex analytic mapping $z^{l}$, for $l$ an integer $\geqq 2$. The multiplicity of this branch point is defined to be $l-1$.

If $F: N \rightarrow M$ is smooth, we define $\partial F=\left.F\right|_{\partial N}$. We say $\partial F$ is regular if $\left.F\right|_{\partial D_{k}}$ is regular for $k=1, \cdots, n$. If $y \in M$ is not on the image of $\partial F$, we define $\operatorname{twn}(\partial F ; y)=\sum_{k=1}^{n} \operatorname{twn}\left(\left.F\right|_{\partial D_{k}} ; y\right)$. We wish to investigate the relationship between twn $(\partial F ; y)$ and the total branchpoint multiplicity at branchpoints of $F$, if $F$ is a branched mapping.

Lemma 1. Let $F: C \rightarrow C$ be the complex map $z^{l}, l \geqq 2$ and let $v$ be a nonzero vector field on $C$, then $\operatorname{ind}_{0} F_{*} v=l-1$.

Proof. Let $\tau=\tau(z)$ be a complex valued function giving the vector field $v$. Identifying $T C$ with $C \times C$, the map $F_{*} v$ is given by $z \rightarrow\left(z^{l}, l z^{l-1} \tau\right)$. Now $\operatorname{ind}_{0} F_{*} v=(1 / 2 \pi) \int_{|z|=1} d \arg l z^{l-1} \tau$. Since $\tau(z) \neq$ 0 for $z \in C, \int_{|z|=1} d \arg \tau=0$. Therefore

$$
\operatorname{ind}_{0} F_{*} v=(1 / 2 \pi) \int_{|z|=1} d \arg l z^{l-1}=l-1,
$$

which completes the proof of the lemma.

TheOREM 3. Suppose $F: N \rightarrow M$ is a branched mapping, $\partial F$ is regular, and $y \in M-F(\partial N)$, then

$$
\operatorname{twn}(\partial F ; y)+I(F, y) \chi(M)=\chi(N)+r
$$

where $r$ is the total branchpoint multiplicity at branchpoints of $F$.

Proof. Let $\left\{x_{1}, \cdots, x_{m}\right\}=B$ be the set of branchpoints of $F$. Let $\left\{x_{m+1}, \cdots, x_{l}\right\}=F^{-1}(y)$. Note that $l-m=I(F, y)$.

Without loss of generality, assume that $y$ is a regular value 
of $F$ and $B \cap F^{-1}(y)=\varnothing$. Let $v_{1}$ and $v_{2}$ be vector fields on $M$ such that $\left(v_{1}, v_{2}\right)$ is positively oriented on $M$ except at $y$, where both vector fields vanish to the order $\chi(M)$. Let $w$ be a vector field on $N$ which defines positive orientation on $\partial N$. Suppose that $w$ vanishes only at $x_{0} \notin B \cup F^{-1}(y)$. Write

$$
F_{*} w(x)=p_{1}(x) v_{1}(f(x))+p_{2}(x) v_{2}(f(x))
$$

where $p=\left(p_{1}, p_{2}\right): N-F^{-1}(y) \rightarrow R^{2}$. Choose disjoint closed coordinate $\operatorname{discs} T_{0}, \cdots, T_{l}$ with $x_{k} \in T_{k}$ for $k=0,1, \cdots, l$.

Since $F$ is regular and preserves orientation except at $x_{1}, \cdots$, $x_{m}$, we have

(a) $\left.\operatorname{deg}(p /|p|)\right|_{\partial T_{0}}=\chi(N)$.

(b) For $k=m+1, \cdots, l,\left.\operatorname{deg}(p /|p|)\right|_{\partial T_{k}}=-\chi(M)$.

Also by Lemma 1 we have

(c) For $k=1, \cdots, m,\left.\operatorname{deg}(p /|p|)\right|_{\partial T_{k}}=r_{k}-1$ where $r_{k}$ is the branchpoint multiplicity at $x_{k}$.

Finally, by definition

(d) $\left.\operatorname{deg}(p /|p|)\right|_{\partial N}=\operatorname{twn}(\partial f ; y)$.

Since $p$ is a smooth map from $N-\bigcup_{k=0}^{l} T_{k}$ into $R^{2}$, we have also $\left.\operatorname{deg}(p /|p|)\right|_{\partial N}=\left.\sum_{k=0}^{l} \operatorname{deg}(p /|p|)\right|_{\partial T_{k}}$. The theorem now follows from Remarks (a), (b), (c), and (d).

Theorem 3 is intended to be a generalization of results of the type stated by Titus [10], Haefliger [4], and Francis [2]. This is illustrated by the following corollaries.

CoROllary 1. If $F: N \rightarrow R^{2}$ is a branched mapping and $\partial F$ is regular, then twn $\partial F=\chi(N)+r$ where $r$ is the total multiplicity at branchpoints of $F$, and twn is the usual tangent winding number for regular curves in the plane.

Proof. Let $M=S^{2}$ in Theorem 3 and identify $R^{2}$ with $S^{2}-\{y\}$. Then $I(F, y)=0$, twn $\partial F=\operatorname{twn}(\partial F ; y)$, and the theorem follows.

COROLLARY 2. If $F: N \rightarrow R^{2}$ is a sense-preserving immersion and $\partial F$ is regular, then twn $\partial F=\chi(N)$.

For information on assembling branched mappings see Francis [2] and Marx [5].

To show how the classical Riemann-Hurwitz theorem follows from Theorem 3 , we prove

Corollary 3 (Riemann-Hurwitz). If $\widetilde{F}: \widetilde{N} \rightarrow M$ is a branched 
mapping, where $\tilde{N}$ and $M$ are compact oriented 2-manifolds, then $\chi(\tilde{N})+r=(\operatorname{deg} \tilde{F}) \chi(M)$.

Proof. Let $y$ be a regular value of $\widetilde{F}$ and $D$ a sufficiently small open disc containing $y$ such that $\widetilde{F}^{-1}(D)$ consists of $\operatorname{deg} \widetilde{F}$ disjoint discs $D_{j}$. Let $N=\widetilde{N}-\cup D_{j}$ and $F=\left.\widetilde{F}\right|_{N}$. Now twn $\left(\left.F\right|_{\partial D_{j}}\right.$; $y)=\chi(M)-1$ for $j=1, \cdots, \operatorname{deg} \widetilde{F}$ and $I(F ; y)=0$. Therefore Theorem 3 gives

$$
(\operatorname{deg} \tilde{F})(\chi(M)-1)=\chi(N)+r=\chi(\tilde{N})-\operatorname{deg} \widetilde{F}+r
$$

and the conclusion follows.

\section{REFERENCES}

1. D. R. J. Chillingworth, Winding numbers on surfaces I, Math. Ann., 196 (1972), 218-249.

2. G. K. Francis, Assembling compact Riemann surfaces with given boundary curves and branch points on the sphere, Illinois J. Math., 20 (1976), 198-217.

3. V. Guillemin and A. Pollack, Differential Topology, Prentice-Hall, Inc., Englewood Cliffs, New Jersey, 1974.

4. A. Haefliger, Quelques remarques sur les applications diffèrentiable d'une surface dans le plan, Ann. Inst. Fourier, 10 (1960), 47-60.

5. M. L. Marx, Extensions of normal immersions of $S^{1}$ into $R^{2}$, Trans. Amer. Math. Soc., 187 (1974), 309-326.

6. J. R. Quine, Homotopies and intersection sequences, Pacific J. Math., (to appear).

7. - A global theorem for singularities of maps between oriented 2-manifolds, (to appear), Trans. Amer. Math. Soc.

8. B. L. Reinhart, The winding number on two manifolds, Ann. Inst. Fourier, 10 (1960), 271-283.

9. S. Smale, Regular curves on Riemannian manifolds, Trans. Amer. Math. Soc., 87 (1958), 492-512.

10. C. J. Titus, Extensions through codimension one to sense preserving mappings, Ann. Inst. Fourier, 23 (1973), 215-227.

11. H. Whitney, On regular closed curves in the plane, Compositio Math., 4 (1937), 276-286.

12. - On singularities of mappings of Euclidean spaces, I, mappings of the plane into the plane, Ann. of Math., 62 (1955), 374-410.

Received October 1, 1976

Florida State University

Tallahassee, FL 32306 



\section{PACIFIC JOURNAL OF MATHEMATICS}

EDITORS

RICHARD ARENS (Managing Editor)

University of California

Los Angeles, California 90024

C. W. Curtis

University of Oregon

Eugene, OR 97403

C. C. MOORE

J. DUGUNDJI

Department of Mathematics University of Southern Californa Los Angeles, California 90007

R. Finn AND J. Milgram Stanford University Stanford, California 94305

University of California

Berkeley, CA 94720

\section{ASSOCIATE EDITORS}

E. F. BeCKENBACH

B. H. NeUMANN

F. WOLF

K. YosHida

\section{SUPPORTING INSTITUTIONS}

UNIVERSITY OF BRITISH COLUMBIA UNIVERSITY OF SOUTHERN CALIFORNIA CALIFORNIA INSTITUTE OF TECHNOLOGY UNIVERSITY OF CALIFORNIA MONTANA STATE UNIVERSITY UNIVERSITY OF NEVADA, RENO STANFORD UNIVERSITY UNIVERSITY OF TOKYO UNIVERSITY OF UTAH NEW MEXICO STATE UNIVERSITY WASHINGTON STATE UNIVERSITY OREGON STATE UNIVERSITY UNIVERSITY OF WASHINGTON UNIVERSITY OF OREGON OSAKA UNIVERSITY 


\section{Pacific Journal of Mathematics \\ Vol. 73, No. 1 \\ March, 1977}

Thomas Robert Berger, Hall-Higman type theorems. $V \ldots \ldots \ldots \ldots \ldots \ldots \ldots$

Frank Peter Anthony Cass and Billy E. Rhoades, Mercerian theorems via

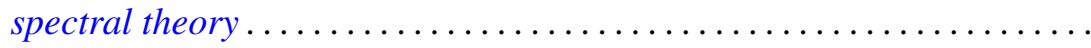

Morris Leroy Eaton and Michael David Perlman, Generating $\mathrm{O}(n)$ with

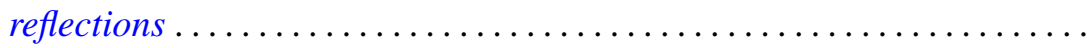

Frank John Forelli, Jr., A necessary condition on the extreme points of a

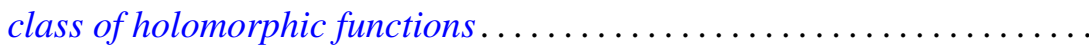

Melvin F. Janowitz, Complemented congruences on complemented

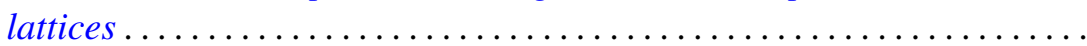

Maria M. Klawe, Semidirect product of semigroups in relation to amenability, cancellation properties, and strong $F \phi$ lner conditions....

Theodore Willis Laetsch, Normal cones, barrier cones, and the "spherical image" of convex surfaces in locally convex spaces ................

Chao-Chu Liang, Involutions fixing codimension two knots.............

Joyce Longman, On generalizations of alternative algebras .............

Giancarlo Mauceri, Square integrable representations and the Fourier

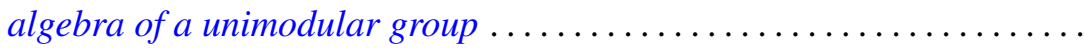

J. Marshall Osborn, Lie algebras with descending chain condition...

John Robert Quine, Jr., Tangent winding numbers and branched

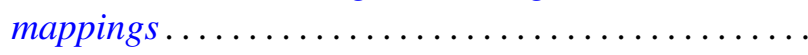

Louis Jackson Ratliff, Jr. and David Eugene Rush, Notes on ideal covers and associated primes .

H. B. Reiter and N. Stavrakas, On the compactness of the hyperspace of faces.

Walter Roth, A general Rudin-Carlson theorem in Banach-spaces ..

Mark Andrew Smith, Products of Banach spaces that are uniformly rotund in every direction.

Roger R. Smith, The R-Borel structure on a Choquet simplex ...

Gerald Stoller, The convergence-preserving rearrangements of real infinite series. ...

Graham H. Toomer, Generalized homotopy excision theorems modulo a Serre class of nilpotent groups...

Norris Freeman Weaver, Dehn's construction and the Poincaré conjecture....

Steven Howard Weintraub, Topological realization of equivariant intersection forms... 